\title{
Extended Model of Stock Price Behaviour
}

\author{
Nico Koning ${ }^{*}$, Daniel T. Cassidy ${ }^{2}$, Rachid Ouyed ${ }^{1}$ \\ ${ }^{1}$ Department of Physics and Astronomy, University of Calgary, Calgary, Canada \\ ${ }^{2}$ Department of Engineering Physics, McMaster University, Hamilton, Canada \\ Email: ^nakoning@ucalgary.ca
}

How to cite this paper: Koning, N., Cassidy, D.T. and Ouyed, R. (2018) Extended Model of Stock Price Behaviour. Journal of Mathematical Finance, 8, 1-13. https://doi.org/10.4236/jmf.2018.81001

Received: October 30, 2017

Accepted: January 16, 2018

Published: January 19, 2018

Copyright $\odot 2018$ by authors and Scientific Research Publishing Inc. This work is licensed under the Creative Commons Attribution International License (CC BY 4.0).

http://creativecommons.org/licenses/by/4.0/

\begin{abstract}
We have developed an extended model for stock price behaviour that is able to accommodate fat-tailed distributions with support as large as $[-\infty, \infty]$. The "homogeneously saturated" (HS) model avoids exponential price changes for large fluctuations by means of a saturation parameter. In the limit where the saturation parameter is zero, the standard model of stock price behaviour (i.e., geometric Brownian motion) is recovered. We compare simulated stock price series generated for both the standard and HS model for the DJIA and five random stocks from the NYSE and NASDAQ exchanges. We find that in all cases, the HS model provides a better fit to the observed price series than the standard model. This has implications to many areas of finance including the Black-Scholes formula for option pricing.
\end{abstract}

\section{Keywords}

Stock Price, Fat-Tail, Brownian Motion, Student's T, Black-Scholes

\section{Introduction}

The standard model of stock price behaviour generates prices through geometric Brownian motion with a deterministic drift rate [1] [2] [3] [4]. This model is used throughout the financial world; most notably in deriving the Black-Scholes formula of option pricing. The standard model for generating stock prices is

$$
\frac{\mathrm{d} S(t)}{\mathrm{d} t}=\alpha S(t)+\sigma S(t) f(t)
$$

where $S(t)$ is the price of the stock at time $t, \alpha$ is the drift rate, $\sigma$ is the volatility of the stock price and $f(t)$ is a zero mean, normally distributed, stochastic, uncorrelated in time, noise driving term. Using $\eta(t)=\alpha+\sigma f(t)$, we can simplify Equation (1) to

$$
\frac{\mathrm{d} S(t)}{\mathrm{d} t}=\eta(t) S(t) .
$$


Integrating Equation (2) gives the predicted price of the stock at a later time $t$ :

$$
S_{s}(t)=S_{0} \mathrm{e}^{\omega(t)}
$$

where the subscript $s$ denotes the standard model, $S_{0}$ is the price of the stock at $t=0$, and for clarity, we have made the substitution

$$
\omega(t)=\int_{0}^{t} \eta\left(t^{\prime}\right) \mathrm{d} t^{\prime}
$$

Equations (3) and (4) suggest that the price of the stock depends exponentially on the integral of the noise driving term, $f(t)$. Since this term is assumed to be normally distributed, the probability of a large price is essentially zero and the predicted price remains bounded. If, however, the underlying noise is not normally distributed, Equation (3) might predict wild price swings that are unrealistic and not observed on the market.

Stock returns are generally assumed to follow a normal distribution, in part owing to mathematical simplicity. It has been known for some time, however, that this assumption is not supported by actual stock prices (e.g., [5] [6] [7]). For example, daily returns of the DJIA and the S\&P 500 indices are described by a fat-tailed distribution [3] [8]. Prices predicted by the standard model, using a normal distribution as the noise driving term, will therefore be inaccurate and simply substituting a fat-tailed distribution will permit the infinite prices mentioned above.

A simulation of stock prices using Equation (2) and drawing $\eta(t)$ from different distributions is given in Figure 1. The fat-tailed distribution used in

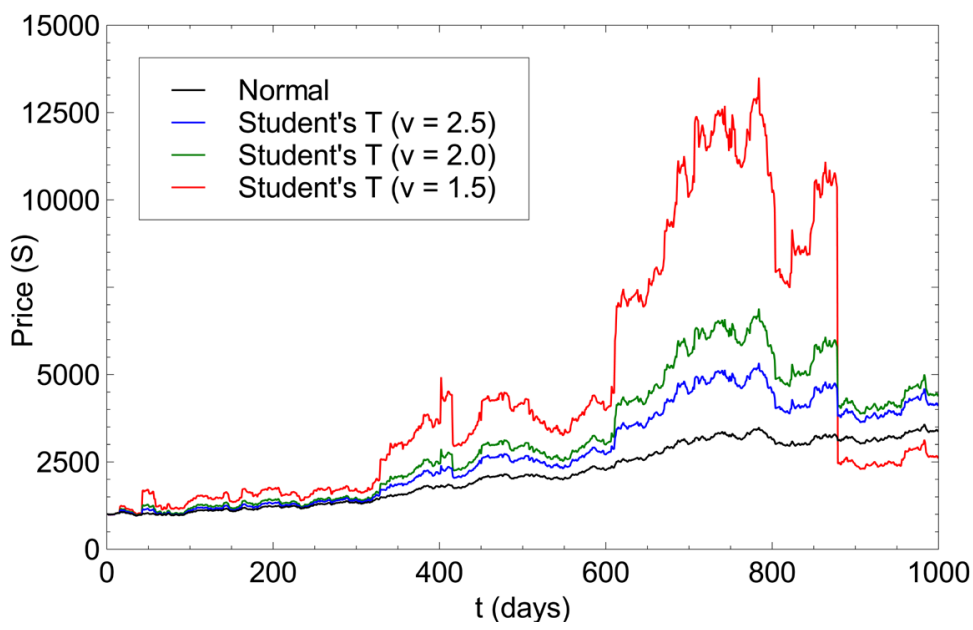

Figure 1. Simulated stock price over 1000 days using the standard model, Equation (2), with $S_{0}=1000, t=1$ day, and $\eta(1)$ drawn from different distributions but for the same sequence of pseudo random numbers. The black line shows daily increments from a normal distribution with $\sigma=0.01, \mu=0.001$; the blue line shows draws from a Student's T with $v=2.5$; the green line, a Student's T with $v=2.0$; and the red line, a Student's T with $v=1.5$. Each Student's T distribution has $\beta=0.01$ and $\mu=0.001$. It is evident that when the noise used in the standard model is a fat-tailed distribution (e.g., Student's $\mathrm{T})$, the predicted price is subject to large fluctuations. The extent of these fluctuations increases with fatter tails (i.e., smaller $v$ ). 
this comparison is the Student's T distribution (see Appendix A), where the "fatness" of the tails is governed by the shape parameter $v$. It is clear from Figure 1 that when a fat-tailed distribution is used in the standard model, large price fluctuations can result.

There exist several approaches to pricing stocks when the noise driving term is a fat-tailed distribution. One approach is to modify the tails of the distribution such that the contributions far into the tails are negligible while not affecting significantly the central portion of the distribution, which fits well the observed data (e.g. [9] [10] [11] [12] [13]). Capping the value of the stock [8] [14], or truncating the distribution [13] are alternative possibilities.

A different approach to pricing stocks when the underlying distribution is fat-tailed is to allow for saturation of the stock price by depletion of the resource that supports the price (i.e., by depletion of the reservoir of money that is available to purchase the stock). This is the approach we investigate in this paper. This "homogeneously saturated" (HS) model for the price of a stock is constructed and compared to the standard model to gain insight into the pricing of financial assets when the underlying distribution is fat-tailed.

\section{Homogeneously Saturated (HS) Model}

The aim of this section is to develop a pricing model which can handle fat-tailed distributions as the noise driving term. Let $M(t)$ be the amount of money available in a reservoir to buy the stock, $N$ the rate at which money is added to the reservoir, $\gamma S(t) M(t)$ the rate at which money is removed from the reservoir due to the purchase of the stock, and $\zeta M(t)$ the rate at which money is removed from the reservoir due to the purchase of other goods. We then have

$$
\frac{\mathrm{d}}{\mathrm{d} t} M(t)=N-\gamma S(t) M(t)-\zeta M(t)+\delta f(t)
$$

where $\delta f(t)$ is a noise driving term. It is interesting to note that the noise in the homogeneously saturated model is ascribed to fluctuations in the amount of money available to invest in the stock. All parameters $(\zeta, N, \gamma)$ in Equation (5) have a time dependence, but it is assumed that these parameters vary slowly enough that they can be treated as constants. As well, we assume the system is in steady state, such that $\frac{\mathrm{d}}{\mathrm{d} t} M(t)=0$, leading to

$$
M(t)=\frac{\alpha+\rho f(t)}{1+\lambda S(t)}
$$

where we have made the substitutions: $\alpha=N / \zeta, \rho=\delta / \zeta$ and $\lambda=\gamma / \zeta$.

The next step in our derivation is inspired from laser physics, where coupled rate equations are used to describe the interaction between the laser output (analogous to $S(t)$ in our case) and the inversion (analogous to $M(t)$ in our case) [15]. The saturation of the inversion in a laser enforces conservation of energy, thereby forcing the output to be finite and to track the input; a trait we 
desire in our model. Therefore coupling $S(t)$ to $M(t)$ will allow for saturation of the stock price such that it cannot continually increase exponentially as allowed by the standard model. We therefore make the following assumption:

$$
\frac{\mathrm{d}}{\mathrm{d} t} S(t)=M(t) S(t) \text {. }
$$

The validity of Equation (7) will be determined by how well it fits the available data. Using Equation (6) in (7) gives

$$
\frac{\mathrm{d}}{\mathrm{d} t} S(t)=\frac{\alpha S(t)+\rho f(t) S(t)}{1+\lambda S(t)} .
$$

Making the substitution $\eta(t)=\alpha+\rho f(t)$ we have

$$
\frac{\mathrm{d}}{\mathrm{d} t} S(t)=\frac{\eta(t) S(t)}{1+\lambda S(t)} \text {. }
$$

The price of the stock in our HS model is therefore

$$
S(t)=\frac{S_{0} \mathrm{e}^{\omega(t)}}{\mathrm{e}^{\lambda\left(S(t)-S_{0}\right)}}=\frac{S_{S}(t)}{\mathrm{e}^{\lambda\left(S(t)-S_{0}\right)}} .
$$

The HS model is similar in form to the standard model, Equation (3), except for the $\mathrm{e}^{\lambda\left(S(t)-S_{0}\right)}$ term in the denominator of the former. Indeed, when $\lambda=0$ the HS model reduces to the standard model. The $S(t)$ dependency in the denominator of Equation (10) effectively saturates the price of the stock; without it wealth would not be conserved and $S(t)$ could continually increase exponentially with time.

To demonstrate the behaviour of the saturation parameter, we generate simulated prices over a 1000 day period using the HS model, Equation (9), by adding different and independent one day solutions for $S(1)$ for each $t$ to obtain the price for the next day, i.e., $S(t+1)=S(t)+S_{t+1}(1)$, for $t=0, \cdots, 1000$ with $S(0)=1000$ and $S_{t+1}(1)$ being the one day solution over the time interval $t$ to $t+1$. These generated price series are shown in Figure 2 for various values of $\lambda$ and for the same sequence of draws from a pseudo random number generator (PRNG) to determine $\eta_{t}(1)$ and hence $S_{t}(1)$ for each $t . \eta_{t}(1)$ is $\eta(1)$ created using the $t^{\text {th }}$ value from the PRNG sequence for a Student's T distribution with shape parameter $v=2.5$, scale parameter $\beta=0.01$, and drift $\mu=0.001$. When $\lambda$ is zero, the price of the stock increases exponentially with the noise driving term and leads to large price swings. The case $\lambda=0$ is equivalent to the standard model with a fat-tailed Student's $\mathrm{T}$ noise source. When $\lambda>0$, the price of the stock approximately linearly follows the noise; i.e., it becomes saturated. Variations in the price of the stock are increasingly damped with higher values of $\lambda$. See Appendix B for a justification of the approach $S(t+1)=S(t)+S_{t+1}(1)$.

To reiterate, $\lambda$ describes the rate at which money is removed from the money reservoir owing to purchase of the stock. $\lambda=0$ (as in the standard model) means that buying the stock has no effect on the money supply; essen- 
tially the standard model assumes an infinite reservoir of money is available to buy the stock. From Equation (9), it can be observed that the reciprocal of the saturation parameter, $\lambda^{-1}$, has the same units as the stock price $S(t)$. One can identify $\lambda^{-1}$ as the stock price at saturation. When $S(t)=\lambda^{-1}$, the instantaneous rate of change with time of $S(t)$ is one-half of what it would be with $\lambda=0$; see Equation (9). For $S(t) \ll \lambda^{-1}$, the reservoir of money is not depleted (or saturated) by the rate of transactions, and the rate of change of $S(t)$ is similar in magnitude to the standard model. For $S(t) \gg \lambda^{-1}$, the reservoir of money that is available to purchase the stock is saturated (or depleted) by the rate of transactions and the time rate of change of $S(t)$ is greatly diminished.

\section{Results}

The goal in developing the HS model is to provide an extension to the standard model that can accommodate assets whose returns are fat-tail distributed. In this section we compare the HS model to the standard model using real data in an effort to corroborate our claim.

Our metric for how close simulated prices match the observed will be the mean absolute percentage error (MAPE) ([16], e.g.), defined as

$$
M(A, O)=100 \times \frac{1}{n} \sum_{t=1}^{n}\left|\frac{A_{t}-O_{t}}{A_{t}}\right|
$$

where $n$ is the number of simulated days, $A_{t}$ is the observed price on day $t$, and $O_{t}$ is the simulated price on day $t$. Of course since we are drawing randomly from distributions, any given simulated price series will yield a different $M$. We therefore create a set of $k=100,000$ simulated price series for each model and compare the average $M$ from those trials. That is, we compare

$$
M_{\text {avg }, D}(\lambda)=\frac{1}{k} \sum_{i=1}^{k} M\left(\operatorname{SIM}_{D}(\lambda), \text { OBS }\right)
$$

where $D$ is the distribution (either $N$ for normal or $S T$ for Student's T), $\operatorname{SIM}_{D}(\lambda)_{i}$ is a simulated price series obtained using Equation (9) (for a given $\lambda$ and distribution), and OBS is the observed price series.

As an example, we use closing values of the Dow Jones Industrial Average (DJIA) for 1000 days starting on January 12010 as our observed price series. The returns along with the Normal and Student's T best fit are shown in the first panel of Figure 3 and the best fit parameters to the 1000 one-day returns are given in the first row of Table 1. It is clear from both Figure 3 and the $\chi^{2}$ in the table that the Student's $\mathrm{T}$ is the superior fit to the DJIA one-day returns.

We begin by determining the saturation parameter, $\lambda_{0}$, that gives the best fit between the Student's T simulated price series and the observed DJIA price series. We do this by using Equation (12) as a function of $\lambda$ in a standard optimization technique (i.e., Brent's Method [17]). We find that $\lambda_{0}=2.04 \times 10^{-4}$, with $M_{\text {avg }}=7.5 \%$ for the DJIA data. We then proceed to determine 


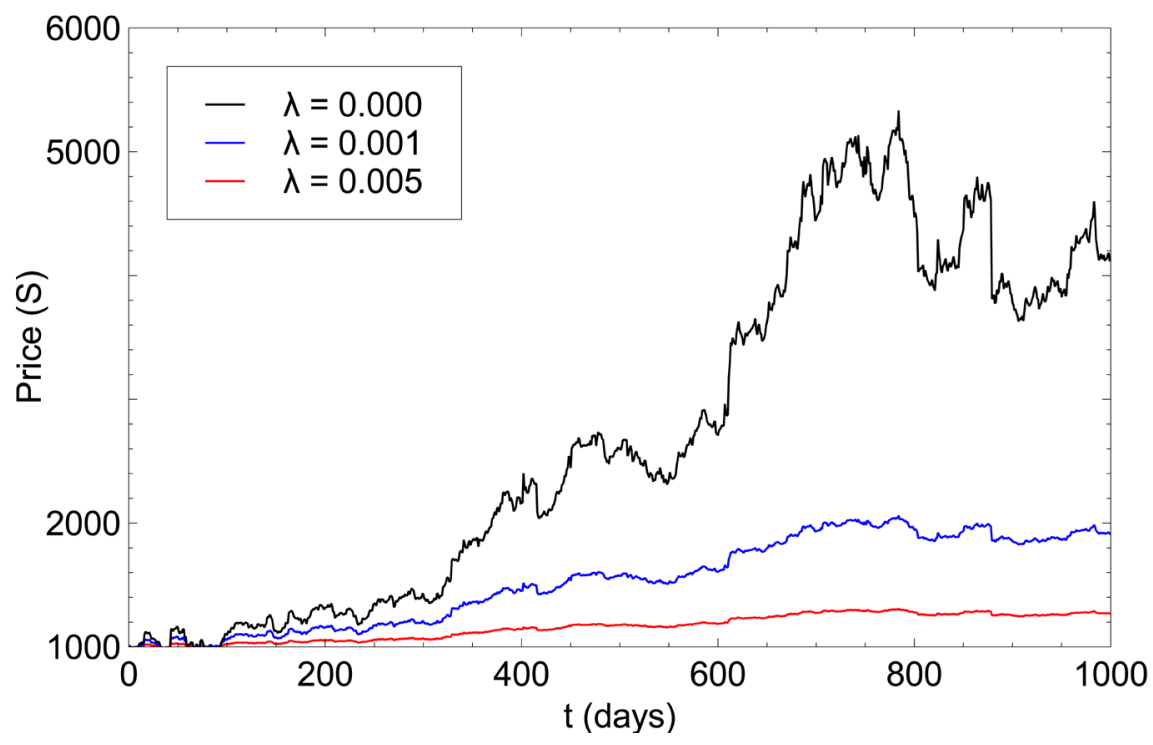

Figure 2. Simulated stock prices $S(t)$ over a 1000 day period using the HS model for $t=1$ day, $S_{0}=1000$, and for various values of $\lambda$. The same sequence of draws from a Student's $\mathrm{T}$ distribution with $v=2.5, \beta=0.01$, and $\mu=0.001$ were used to create the $S(t)$ for the different values of $\lambda$. As $\lambda$ increases, the large price swings seen with the standard model $(\lambda=0)$ are suppressed.

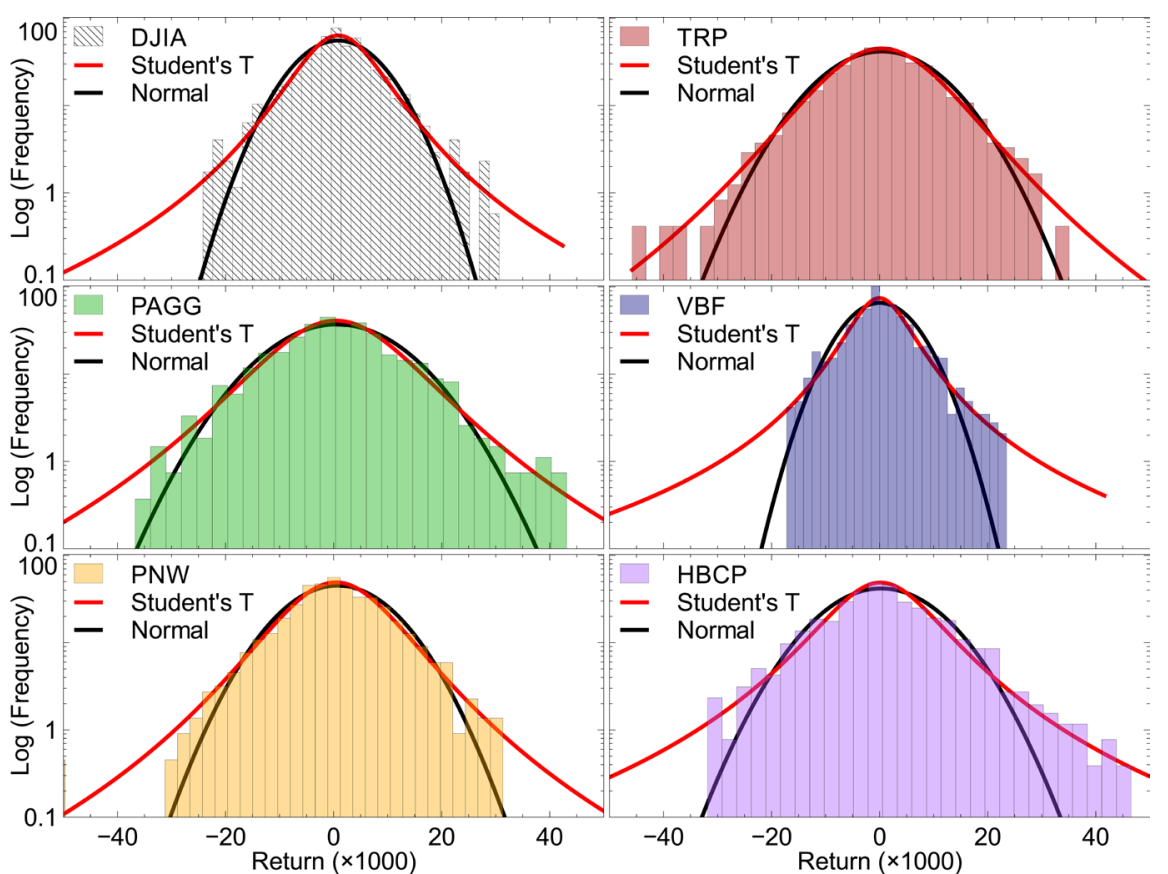

Figure 3. Normal and Student's T fits to daily returns from six different assets. The best fit parameters for each distribution are given in Table 1. In all cases the Student's T provides a superior fit.

- $M_{\text {avg, } N}(0)$ (i.e., standard model, Equation (9) with $\lambda=0$ and $\eta$ drawn from the normal distribution with parameters given in Table 1).

- $M_{\text {avg,ST }}(0)$ (i.e., Student's T distribution directly inserted in the standard 
Table 1. Best fit parameters for Normal and Student's T distributions to one-day returns with \pm uncertainties (confidence level $=0.95$ ) in parentheses. Each stock data set starts on January 12010 and runs for 1000 days. The reduced $\chi^{2}$ for each fit is also given. $\beta$ is the scale parameter for the Student's T distribution; see Appendix A.

\begin{tabular}{cccccccc}
\hline \multicolumn{3}{c}{ Normal } & \multicolumn{5}{c}{ Student's T } \\
\hline & $\sigma\left(\times 10^{3}\right)$ & $\mu\left(\times 10^{3}\right)$ & $\chi^{2}$ & $v$ & $\beta\left(\times 10^{3}\right)$ & $\mu\left(\times 10^{3}\right)$ & $\chi^{2}$ \\
\hline DJIA & $7.18(0.82)$ & $0.82(0.69)$ & 3.28 & $2.65(1.55)$ & $5.72(0.79)$ & $0.85(0.85)$ & 1.81 \\
TRP & $9.56(0.69)$ & $0.43(0.56)$ & 1.23 & $4.99(2.34)$ & $8.40(0.59)$ & $0.33(0.48)$ & 0.57 \\
PAGG & $10.75(1.05)$ & $0.58(0.85)$ & 2.31 & $3.87(2.70)$ & $9.13(1.13)$ & $0.42(0.90)$ & 1.60 \\
VBF & $6.09(0.80)$ & $0.04(0.71)$ & 4.15 & $1.69(0.95)$ & $4.66(0.74)$ & $-0.04(0.59)$ & 1.96 \\
PNW & $8.86(0.78)$ & $0.71(0.65)$ & 1.88 & $3.88(2.49)$ & $7.63(0.81)$ & $0.46(0.90)$ & 1.15 \\
HBCP & $10.61(1.11)$ & $0.37(0.92)$ & 2.70 & $2.61(1.41)$ & $8.49(1.10)$ & $0.14(0.86)$ & 1.51 \\
\hline
\end{tabular}

model).

- $M_{\text {avg,ST }}\left(\lambda_{0}\right)$ (i.e., HS model, Equation (9) with $\lambda=\lambda_{0}$ and $\eta$ drawn from the Student's T distribution with parameters given in Table 1).

The results for the DJIA are given in the first row of Table 2. There are several things to notice. First, the standard model performs relatively poorly for the DJIA with $M_{\text {avg }}=33 \%$. This can be expected since the daily returns of the DJIA are fit much better by a fat-tailed distribution. Second, when a fat-tailed distribution is substituted into the standard model directly, $M_{\text {avg }}$ is actually larger than with a normal distribution despite the better fit to the one-day returns. This, as we mentioned earlier, is expected due to the large probability of large price swings when directly using a fat-tailed distribution in the standard model. These two points taken together justify the need for a better pricing model that can handle fat-tailed distributions. Third, the HS model with $\lambda=2.04 \times 10^{-4}$, clearly gives superior results to the standard model with an $M_{\text {avg }}$ of only $7.5 \%$.

To test these ideas further, we have randomly selected five stocks from the NYSE and NASDAQ exchanges; TRP, PAGG, VBF, PNW and HBCP. The results from these additional stocks are given in Figure 3, Table 1, and Table 2. Specifically, Figure 3 displays best fits of normal and Student's T distributions to the daily returns for the five random stock selections and for the DJIA. The parameters determined for the best fit distributions are used in the simulations with the standard and HS models for the stock price.

In 4 out of the 6 cases, the standard model performs relatively poorly. The exceptions are TRP with $M_{\text {avg }}=19.3 \%$ and VBF with $M_{\text {avg }}=13.5 \%$. For TRP this is expected since its returns are actually well fit by a normal distribution. VBF, on the other hand, is poorly fit by a normal distribution so its low $M_{\text {avg }}$ is surprising.

In all cases, except marginally PNW, simply substituting a fat-tailed distribution into the standard model results in a higher $M_{\text {avg }}$ than the standard model. This, as we mentioned previously, is due to the large price swings inherent 
Table 2. Comparison between simulated and observed price series for different models. The average MAPE (Equation (12)) generated from 100,000 simulated price series and the observed price series (see Figure 5) for various assets are given. $M_{\text {avg }, N}(0)$ represents the standard model (i.e., Equation (9) with $\lambda=0$ and $\eta$ drawn from the normal distribution), $M_{\text {avg, ST }}(0)$ Equation (9) with $\lambda=0$ and $\eta$ drawn from the Student's T distribution, $M_{\mathrm{avg}, S T}\left(\lambda_{0}\right)$ the HS model with $\lambda=\lambda_{0}$ and $\eta$ drawn from the Student's T distribution. The best fit parameters used for each distribution are given in Table 1 . The minimum and maximum $M$ are given in parenthesis.

\begin{tabular}{ccccc}
\hline Stock & $\lambda_{0}$ & $M_{\text {avg, } N}(0)$ & $M_{\text {avg, }, S T}(0)$ & $M_{\text {avg, ST }}\left(\lambda_{0}\right)$ \\
\hline DJIA & 0.000204 & $33.6(3.3,196.0)$ & $42.4(4.3,118.2)$ & $7.5(2.7,177.6)$ \\
TRP & 0.037544 & $19.3(4.0,155.3)$ & $20.3(4.2,187.7)$ & $11.9(2.9,38.7)$ \\
PAGG & 0.266817 & $34.4(4.5,271.2)$ & $31.3(5.4,331.1)$ & $8.0(5.8,17.1)$ \\
VBF & $0.134320^{1}$ & $13.5(3.1,94.4)$ & $42.1(4.7,500699.3)$ & $13.2(2.6,627.2)$ \\
PNW & 0.022735 & $20.5(3.3,166.1)$ & $20.2(3.8,200.8)$ & $13.3(2.7,48.2)$ \\
HBCP & 0.157830 & $19.4(4.0,144.0)$ & $28.8(5.2,991.1)$ & $19.1(2.9,270.8)$ \\
\hline
\end{tabular}

in fat-tailed distributions, and is the motivation for this paper.

Finally in all 6 cases, the HS model performs better than the standard model at predicting future stock prices. The most surprising result here is that for VBF the HS model ( $M_{\text {avg }}=13.2 \%$ ) outperforms the standard model ( $M_{\text {avg }}=13.5 \%$ ) by only a slight margin. This is unexpected since VBF returns are fit much better by a fat $(v=1.69)$ Student's $\mathrm{T}$ distribution. A clue may lie in the exceptionally high maximum $M$ score for $\mathrm{VBF}(\sim 600 \%)$ indicating that large price fluctuations are still present. This, however, is not nearly as large as the maximum $M$ score of $\sim 500,000 \%$ for a Student's T distribution in the standard model without saturation. This may signal a limitation of the HS model in that although the addition of the saturation parameter, $\lambda$, reduces the effects of large price swings for fat-tailed distributions, it does not eliminate them altogether. This limitation is most pronounced for exceptionally fat-tailed distributions where the probability of large returns is likely.

Figure 4 shows the distribution of 100,000 $M$ values for each stock, with a bin width of $M=1 \%$ for the standard and HS models. In all cases except HBCP, the HS model distribution is thinner and further left than the standard model. This implies that the HS model is more likely to match the observed stock price. VBF and HBCP have nearly overlapping distributions which is affirmed by their similar $M_{\text {avg }}$ scores between the standard and HS models. Figure 5 shows example price series for each stock taken from those that make up the peak bin of the distribution in Figure 4. This gives an indication of how well each model may be expected to perform.

\footnotetext{
${ }^{1} M_{\text {avg }, S T}\left(\lambda_{0}\right)$ does not yield a minimum value for the VBF data. This is because the low $v$ parameter for $\mathrm{VBF}$ ensures that extreme price swings are realized even when the saturation parameter is large (see text). Essentially $\lambda=\infty$, a straight line, gives the lowest average. The minimum $M$ was used in the optimization to find $\lambda_{0}$ for this case only.
} 


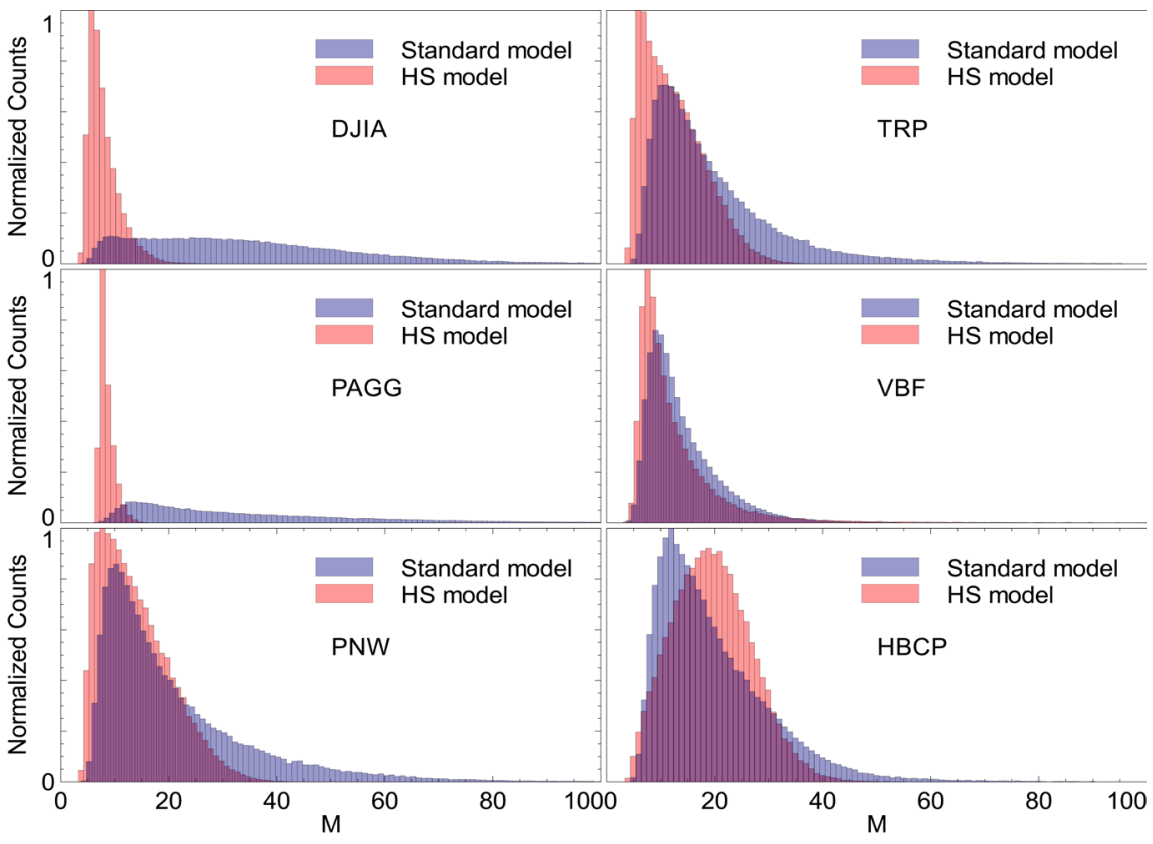

Figure 4. Distribution of MAPE scores using both the standard and HS model for six different assets. In all cases, except HBCP, the HS model has a narrower distribution with a lower peak MAPE score.

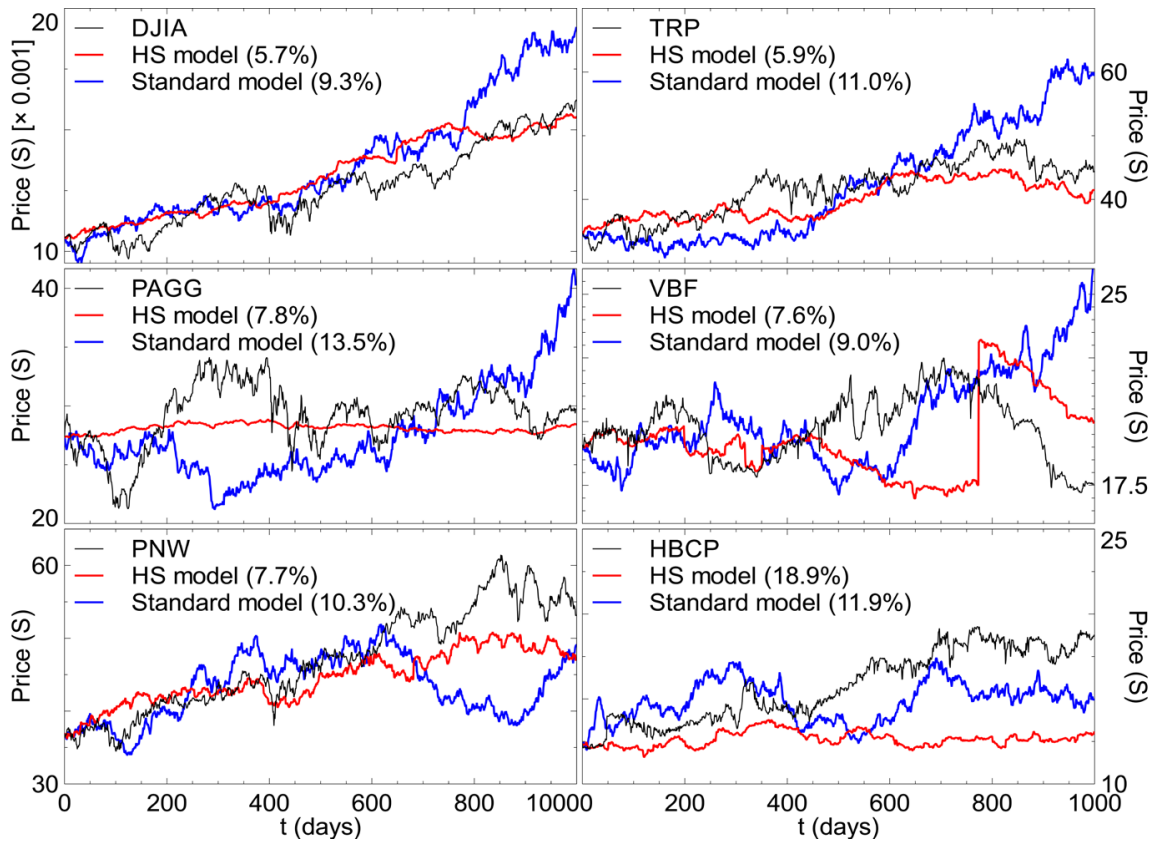

Figure 5. Examples of price series using both the standard and HS model. The price series for each model was chosen randomly from those that make up the peak bin (MAPE score given in parentheses) of their respective distributions shown in Figure 4. The observed price series for each asset is shown in black.

\section{Conclusions}

The standard model, Equation (1), is the most popular model for stock price behaviour. It has wide reaching influence, most notably in the development of 
the Black-Scholes formula for option pricing ([1], e.g.). A primary assumption of the standard model is that daily stock returns follow a normal distribution; an assumption unfounded in the actual market. Fat-tailed distributions fit the returns much better, but when the support for the fat-tailed distributions is assumed to be $[-\infty,+\infty]$ and these fat-tailed distributions are used in the standard model, the standard model allows for extreme (infinite) price swings that are not observed. In this paper we have developed a model for stock price behaviour that adequately allows for fat-tailed distributions with support of $[-\infty,+\infty]$. Using data from the DJIA and five random stocks from the NYSE and NASDAQ exchanges, we have shown/reaffirmed:

- Daily stock returns are indeed better fitted by a fat-tailed distribution (Student's $\mathrm{T}$ in this case) than by a normal (Gaussian) distribution.

- The standard model, in most cases, performs poorly when predicting future stock prices.

- Fat-tailed distributions, when substituted directly into the standard model $(\lambda=0)$, perform poorly when predicting future stock prices.

- A non-zero saturation parameter, $\lambda$, when added to the standard model is able to effectively suppress large price swings inherent in fat-tailed distributions for daily returns. $\lambda$ represents the fraction of money removed from the reservoir due to the purchase of the stock. In the standard model $\lambda=0$ which implies an infinite amount of money available for the purchase of the stock.

- The HS model $(\lambda \neq 0)$ developed in this paper is able to incorporate fat-tailed distributed returns, and consistently outperforms the standard model when predicting future stock prices.

We have shown that a model for stock price behaviour using fat-tailed distributions with support as large as $[-\infty,+\infty]$ for daily returns is attainable. This model is realistic in that it allows for a finite supply of money to be saturated by the net rate of transactions and does not require truncation or capping [8] [9] [13] [14], or modification of the distribution of returns [10] [11] [12] to avoid very large and unobserved stock prices. This homogeneously saturated model, which borrows from laser physics [15], will have widely spread repercussions, most importantly for the Black-Scholes formula for option pricing, which will be investigated in future work. The homogeneously saturated (HS) model requires that a value for the saturation parameter $\lambda$, which is defined in Equation (9), must be estimated from historical data or assumed. The HS model ascribes fluctuations in the price of a stock to fluctuations in the amount of money available to invest in the stock.

\section{Acknowledgements}

This work was funded in part by the Natural Sciences and Engineering Research Council of Canada.

\section{References}

[1] Hull, J.C. (2006) Options, Futures, and Other Derivatives. 6th Edition, Pearson 
Education Inc., New Jersey, 269-272.

[2] McDonald, R.L. (2006) Derivatives Markets. 2nd Edition, Pearson Education Inc., Boston, Ch. 20.

[3] Bouchard, J.-P. and Potters, M. (2003) Theory of Financial Risk and Derivative Pricing. 2nd Edition, Cambridge University Press, Cambridge, Ch. 13. https://doi.org/10.1017/CBO9780511753893

[4] Lax, M., Cai, W. and Xu, M. (2006) Random Processes in Physics and Finance. Oxford University Press, New York, Ch. 16.4. https://doi.org/10.1093/acprof:oso/9780198567769.001.0001

[5] Mandelbrot, B. (1963) The Variation of Certain Speculative Prices. Journal of Business, 36, 394-419. https://doi.org/10.1086/294632

[6] Fama, E. (1965) The Behavior of Stock Market Prices. Journal of Business, 38, 34-105. https://doi.org/10.1086/294743

[7] Clark, P. (1973) A Subordinated Stochastic Process Model with Finite Variance for Speculative Prices. Econometrica, 41, 135-155. https://doi.org/10.2307/1913889

[8] Cassidy, D.T., Hamp, M.J. and Ouyed, R. (2010) Pricing European Options with a log Student's t-Distribution: A Gosset Formula. Physica A, 389, 5736-5748. https://doi.org/10.1016/j.physa.2010.08.037

[9] Cassidy, D.T. (2011) Describing n-Day Returns with Student's t-Distributions. Physica $A$, 390, 2794-2802. https://doi.org/10.1016/j.physa.2011.03.019

[10] Moriconi, L. (2007) Delta Hedged Option Valuation with Underlying Non-Gaussian Returns. Physica A, 380, 343-350. https://doi.org/10.1016/j.physa.2007.01.018

[11] Lim, G.C., Martin, G.M. and Martin, V.L. (2006) Pricing Currency Options in the Presence of Time-Varying Volatility and Non-Normalities. Journal of Multinational Financial Management, 16, 291-314. https://doi.org/10.1016/j.mulfin.2005.08.004

[12] Lye, J.N. and Martin, V.L. (1993) Robust Estimation, Non-Normalities and Generalized Exponential Distributions. Journal of the American Statistical Association, 88, 261-267.

[13] Cassidy, D.T. (2012) Effective Truncation of a Student's t-Distribution by Truncation of the Chi Distribution in a Chi-Normal Mixture. Open Journal of Statistics, 2, 519-525. https://doi.org/10.4236/ojs.2012.25067

[14] Cassidy, D.T., Hamp, M.J. and Ouyed, R. (2013) Log Student's t-Distribution Based Option Sensitivities: Greeks for the Gosset Formulae. Quantitative Finance, 13, 1289-1302. https://doi.org/10.1080/14697688.2012.744087

[15] Cassidy, D.T. (1984) Analytic Description of a Homogeneously Broadened Injection Laser. IEEE Journal of Quantum Electronics, QE-20, 913-918. https://doi.org/10.1109/JQE.1984.1072481

[16] Hyndman, R.J. and Athanasopoulos, G. (2013) Forecasting: Principles and Practice. OTexts, Melbourne, Ch. 2.5.

[17] Brent, R.P. (1973) Algorithms for Minimization without Derivatives. Prentice-Hall, Englewood Cliffs, NJ, Ch. 4. 


\section{Appendix A: Student's T Distribution}

A Student's $\mathrm{T}$ distribution is a fat-tailed distribution with a probability density function

$$
f(t)=\frac{1}{\beta \sqrt{\pi v}} \frac{\Gamma\left(\frac{v+1}{2}\right)}{\Gamma\left(\frac{v}{2}\right)}\left(1+\frac{(t-\mu)^{2}}{v \beta^{2}}\right)^{-\frac{v+1}{2}}
$$

where $\Gamma$ is the gamma function, $\mu$ is the mean, $\beta$ is the scale parameter, and $v$ is the shape parameter. Figure A1 shows the effect of varying $v$ (left panel) and $\beta$ (right panel). The standard deviation of Equation (A.1) is $\sigma=\beta \sqrt{\frac{v}{v-2}}$ for $v>2$.

\section{Appendix B: Additivity of Solutions}

We wish to show that solutions $S(\cdot)$ to the differential Equation (9) have the property $S(t+1)=S(t)+S_{t+1}(1)$.

Add a second argument to $S(t)$ and $w(t)$ to indicate the time origin:

$$
w(a, b)=\int_{a}^{b} \eta(\xi) \mathrm{d} \xi
$$

and

$$
S(a, b)=\frac{S(a, a) \mathrm{e}^{w(a, b)}}{\exp (\lambda(S(a, b)-S(a, a)))} .
$$

$S(a, a)$ equals $S(a)$ and is the value of $S$ at $a . S(a, b)$ is the value of $\left.S(t)\right|_{t=b}$ given the initial value at $t=a$ was $S(a)=S_{a}$.

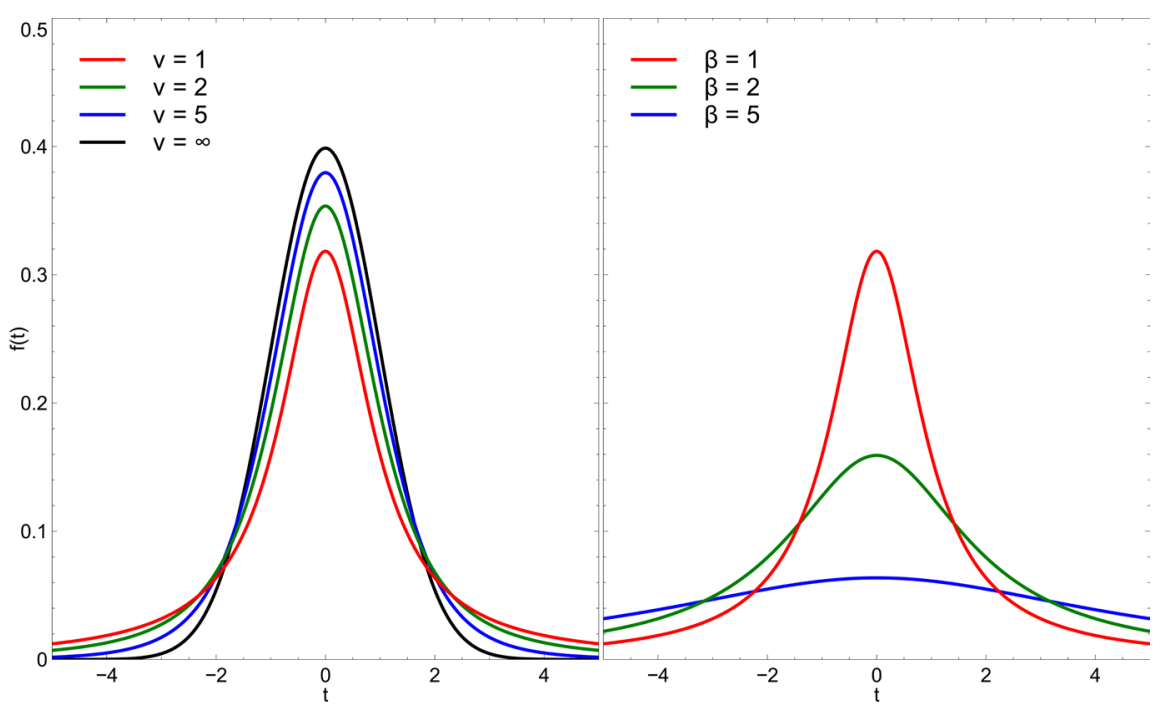

Figure A1. Effect of varying $v$ (left panel, $\beta=1, \mu=0$ ) and $\beta$ (right panel, $v=1$, $\mu=0$ ) on the Student's T distribution (Equation (A.1)). $v=\infty$ corresponds to the normal distribution. 
Start with $S(a, b)+S(b, c)$ and use Equation (B.2) to simplify:

$$
\begin{gathered}
=\frac{S_{a} \mathrm{e}^{w(a, b)}}{\exp \left(\lambda\left(S(a, b)-S_{a}\right)\right)}+\frac{S_{b} \mathrm{e}^{w(b, c)}}{\exp \left(\lambda\left(S(b, c)-S_{b}\right)\right)} \\
=\frac{\exp \left(\lambda\left(S(b, c)-S_{b}\right)\right) S_{a} \mathrm{e}^{w(a, b)}+\exp \left(\lambda\left(S(a, b)-S_{a}\right)\right) S_{b} \mathrm{e}^{w(b, c)}}{\exp \left(\lambda\left(S(a, b)+S(b, c)-\left(S_{a}+S_{b}\right)\right)\right)} \\
=\frac{\frac{S_{b} \mathrm{e}^{w(b, c)}}{S(b, c)} S_{a} \mathrm{e}^{w(a, b)}+\frac{S_{a} \mathrm{e}^{w(a, b)}}{S(a, b)} S_{b} \mathrm{e}^{w(b, c)}}{\exp \left(\lambda\left(S(a, b)+S(b, c)-\left(S_{a}+S_{b}\right)\right)\right)} \\
=\frac{S_{a} S_{b} \mathrm{e}^{w(a, c)}\left(\frac{1}{S(b, c)}+\frac{1}{S(a, b)}\right)}{\exp \left(\lambda\left(S(a, b)+S(b, c)-\left(S_{a}+S_{b}\right)\right)\right)} \\
=\frac{(S(a, b)+S(b, c)) \mathrm{e}^{w(a, c)}\left(\frac{S_{a} S_{b}}{S(a, b) S(b, c)}\right)}{\exp \left(\lambda\left(S(a, b)+S(b, c)-\left(S_{a}+S_{b}\right)\right)\right)} .
\end{gathered}
$$

But $S_{b}=S(a, b)$, by definition, since $S_{b}$ is the value of $S(t)$ at time $t=b$, $a<b<c$, given that the initial value for the time series is $S_{a}$. Then,

$$
\begin{gathered}
S(a, b)+S(b, c) \\
=\frac{(S(a, b)+S(b, c)) \mathrm{e}^{w(a, c)}\left(\frac{S_{a}}{S(b, c)}\right)}{\exp \left(\lambda\left(S(b, c)-S_{a}\right)\right)}
\end{gathered}
$$

or

$$
S(b, c)=\frac{S_{a} \mathrm{e}^{w(a, c)}}{\exp \left(\lambda\left(S(b, c)-S_{a}\right)\right)} .
$$

$S(b, c)$ is the value of $\left.S(t)\right|_{t=c}$ given a value of $S_{b}$ at time $t=b$. The interim value $b$ does not appear in Equation (B.11). If one replaces $S(b, c)$ in Equation (B.11) with $S(a, c)=S_{c}$, since the initial value for the time series is $S_{a}$, then one has

$$
S(a, b)+S(b, c)=S(a, c)=S_{c}=\frac{S_{a} \mathrm{e}^{w(a, c)}}{\exp \left(\lambda\left(S_{c}-S_{a}\right)\right)} .
$$

This completes the demonstration and illustrates a useful property of the homogeneously saturated solution. It is possible to simulate a time series by adding increments to the series or by solving for the value over the full time interval. If one chooses to add increments, then one must be careful to use the appropriate initial condition for the increment. This appropriate initial condition is the value just before the increment starts. 